\title{
A Mechanistic Study of the Influence of Graphene Curvature on the Rate of High-Temperature Oxidation by Molecular Oxygen
}

\author{
Ravi Singh and Michael Frenklach* \\ Department of Mechanical Engineering, University of California at Berkeley, Berkeley, \\ California, 94720-1740, USA
}

*Corresponding Author Email: frenklach@berkeley.edu

\begin{abstract}
Kinetic Monte-Carlo (KMC) simulations of the evolution of a graphene sheet in combustion-like environments were performed using a set of newly established elementary reactions of graphene-edge oxidation by $\mathrm{O}_{2}$. The $\mathrm{KMC}$ results revealed two principal pathways for an oxyradical site: oxidation and regeneration of an aromatic radical site. The two pathways compete for oxyradicals, but exclusion of the regeneration pathway does not significantly affect the oxidation rate. Examination of the morphology of the evolving graphene edges indicated that the addition of $\mathrm{O}_{2}$ promotes the formation of five-member rings embedded in the graphene. The overall oxidation rate of the graphene was computed to be time dependent, with reactivity decreasing over time as the ratio of reactive edge sites decreases relative to the number of basalplane carbon atoms. At the same time, the oxidation rate was found to be higher for graphene with a higher initial curvature. Both results are in accord with experimental observations. Analysis showed that distinct aspects of graphene-edge morphology are responsible for curvature either raising or reducing the oxidative reactivity of the graphene edge.
\end{abstract}

\section{Introduction}

Oxidation of carbon by molecular oxygen has been studied for a long time, in various disciplines and contexts. One of the primary interests is mechanistic understanding of the process. It is not straightforward to decipher experimental observations into mechanistic details, and theoretical tools provide increasingly reliable approaches in this direction. Formation of carbon at high temperatures, such as soot formation in flames, is especially amenable to theoretical studies. Indeed, the overall reaction time at high temperatures is on the order of milliseconds and hence only fast reactions should be accounted for. Furthermore, at these conditions the gas-surface reactions follow the direct, Eley-Rideal mechanism [1] that allows a simpler theoretical treatment, as compared to the Langmuir-Hinshelwood [1, 2] and mobile-precursor [3, 4] mechanisms at lower temperatures that increase the complexity of molecular interactions and enlarge the number of possible reactions. The past few decades of theoretical studies of high- 
temperature carbon growth have brought us to mechanistic understanding of the process [5] that is being currently exploited not only for combustion soot [6,7] but also for carbon-black processes [8] as well as gas-phase synthesis of graphene [9]. These accomplishments motivate employing a similar strategy for unravelling details of carbon oxidation, and hence we begin with soot oxidation at high temperatures.

Oxidation of soot by molecular oxygen has received substantial attention over the years (see, e.g., a recent review [10] and accounts given in recent articles [11-16]). Early conception was based on an empirical model of Nagle and Strickland-Constable (NSC) [17], who represented the carbon surface by two empirical sites: less reactive and more reactive. In the early 1990's, Frenklach and co-workers $[5,18,19]$ suggested a physically-resolved model, postulating that oxidation by molecular oxygen occurs on radical sites of aromatic molecules comprising a soot particle, i.e.,

$$
\mathrm{C}_{\text {surface }} \bullet+\mathrm{O}_{2} \rightarrow \text { products }
$$

The rate coefficient of this reaction was assigned by analogy to phenyl oxidation [20]. This simple, one-step kinetic model has been broadly employed in numerical studies of soot formation in hydrocarbon flames.

While the simple reaction model based on chemical analogy, for both surface oxidation and growth, was successful in achieving an order-of-magnitude agreement with experiment $[5,19$, 21, 22], the follow-up research revealed richer chemistry of the growth (see, e.g., [23-31] and references cited therein). One of the main features is surface migration of five-membered rings along zigzag edges of aromatics $[23,26]$. Being in itself intriguing, this process opens numerous reaction possibilities [26, 27, 29-31]. Modeling that included all such identified different reactions exhibited curving of the initially planar aromatics [29]. Recent experimental studies 
suggested a substantial dependence of soot oxidation rate on soot particle curvature [11]. Clearly, a simple model such as (1) is insufficient to explain this observation.

A more detailed look at soot and carbon oxidation has been given within the past decade (see, e.g., $[14,15,32-42]$ and references cited therein). Some of the recent interest is motivated by the use of filters to capture soot from diesel engines and their regeneration through oxidation of the captured soot $[15,43]$. The attention of the present study is on oxidation of soot at high temperatures, i.e., at conditions realized in flames of hydrocarbon fuels and inside combustion engines. Here we explore the kinetic implications of a newly developed detailed reaction mechanism for these conditions [40] by performing sterically-resolved kinetic Monte-Carlo (KMC) simulations of graphene-sheet evolution in $\mathrm{O}_{2}$-rich environments. Our ultimate goal is to compare the prediction of theory with available experimental data. Here we focus on examining mechanistic factors affecting such comparison.

The central issue investigated in the present study is the role played by graphene-sheet curvature in oxidation. Performing initial KMC simulations [40] we noticed deceleration of oxidation with reaction time, in accord with experimental observations of Vander Wal et al. [12] who found reactivity to decrease as oxidation progresses. The site-resolved modeling [40] identified the cause for the decrease in the oxidation rate to be accumulation of five-member rings in the graphene edge, seemingly in contrast with the earlier study of Vander Wal et al. [11] observing that increased curvature leads to increased, not decreased reactivity. The present analysis resolves this apparent conflict. 


\section{Methodology}

The KMC methodology followed that used previously [29, 40, 44]. Briefly, the KMC simulations tracked a single graphene "molecule" evolving in a flame-like environment, but maintaining the gas phase in a constant state, i.e., at constant values of temperature, pressure, and species mole fractions, which allowed us to investigate unambiguously the influence of individual factors on the simulation outcome. At each time step, a reaction event was selected stochastically and then applied.

The processes of surface growth and oxidation were modeled as a Markovian sequence of reaction events. There are two types of reactions that comprise the present model: bimolecular reactions between the gaseous species and surface sites, and unimolecular "decompositions" of surface species. All stochastic events were treated as first-order processes, with the respective per-site rates. The second-order reactions produced pseudo-first-order rate constants through multiplication of the gas-surface rate coefficient by the concentration of the gaseous reactant. The selection of the reaction event and specific graphene-edge site was done by application of the Gillespie algorithm $[45,46]$ adapted for surface processes [47, 48]. Briefly, given an instant of a current reaction event, $t_{n}$, the time of the next reaction event to occur at substrate site $i$ is $t_{n+1, i}=t_{n}-(\ln u) / k_{\text {total }, i}$, where $u$ is a random number distributed uniformly from 0 to 1 and $k_{\text {total }, i}=\Sigma_{j} k_{j, i}$ is the sum of the per-site rates of reactions possible at site $i$. The smallest among the $t_{n+1, i}$ values, computed for all surface reaction sites, becomes the time instant of the next reaction event, $t_{n+1}$, and the particular reaction to occur at that time is chosen according its probability $p_{j, i}=k_{j, i} / k_{\mathrm{total}, i}$ upon drawing another random number $u$. Once a reaction event is implemented, the process repeats itself. 
The set of surface reactions employed in the KMC model contains 45 growth and 45 oxidation reactions. The surface growth reactions and their corresponding rate coefficients are taken from Whitesides and Frenklach $[29,44]$ and 45 of the surface oxidation reactions and their corresponding rate coefficients are from our recent study [40]. This reaction mechanism is constructed for high temperatures, $1500-2500 \mathrm{~K}$; in doing so, it has been presumed that active soot-particle surface is comprised of $\mathrm{H}$-saturated graphene edges and an active, reactive site for the $\mathrm{O}_{2}$ attack is an aromatic radical, created by $\mathrm{H}$-atom abstraction $[5,19]$. At high temperatures and gaseous environments containing hydrogen, the probability of having two adjacent radical sites is relatively low and hence we neglected reactions occurring on such diradical sites. Furthermore, considering the millisecond time scale of the high-temperature processes, we neglected slow reactions, such as edge reconstruction and nascent site deactivation [14, 49]. With these considerations, the primary product of carbon oxidation is $\mathrm{CO}[15,39-41]$ and the $\mathrm{O}$ atoms released in the surface reactions enter into the gas phase. The gaseous concentration of $\mathrm{O}$ atoms and their contribution to soot oxidation is relatively low in flame environments [50,51], as compared to $\mathrm{OH}$ and $\mathrm{O}_{2}$, and hence the oxidative attack of graphene carbons by $\mathrm{O}$ atoms were not included in the present study. Also, the present simulations consider evolution of a single graphene "flake", and hence no particle-dynamics processes, such as oxidation-induced particle fragmentation [51, 52], were included in the model. The reaction mechanism employed in the present study is provided in Table S1 of the Supplementary Data.

To properly account for the evolving curvature, the KMC model was coupled to molecularmechanics geometry optimization using the MM3 potential [53] with the TINKER molecular mechanics package [54]. The MM3 potential has been found to produce geometries for fullerenes in good agreement with the ab initio Hartree-Fock method [55] as well as 
experimental and DFT results for fullerenic fragments [56]. Optimization of the structure geometry was performed after each structure-changing event. The geometry optimization step properly accounted for substrate curvature while maintaining physically accurate bonding and geometric configuration of the evolving structures. A check was made after each geometry optimization to ensure that the geometry produced by the molecular mechanics code was consistent with the bonding implied by the KMC model. Specifically, the code tested if the geometry optimization left all carbon atoms within a specified distance of the other atoms to which they were bonded in the KMC description. If any two carbon bonds were found to be separated by more than two angstroms, the simulation was ended.

The evolution of the graphene structure, referred to hereafter as substrate, was monitored by computing the numbers of reaction events, instantaneous structure size, and its five-member ring fraction, $f_{\mathrm{R} 5}$, the latter defined as [44]

$$
f_{\mathrm{R} 5}=\frac{32}{12} \frac{N_{\mathrm{R} 5}}{N_{\mathrm{R} 5}+N_{\mathrm{R} 6}},
$$

where $N_{\mathrm{R} 5}$ and $N_{\mathrm{R} 6}$ are the number of five- and six-member rings, respectively, and the 32/12 coefficient normalizes Eq. (2) to have $f_{\mathrm{R} 5}=1$ for buckminsterfullerene, $\mathrm{C}_{60}$.

To collect better statistics, simulations for each set of initial conditions were repeated 100 times, each with a different starting random seed. The results reported in this work are averages over these ensembles of simulations. In the previous study [40] we repeated the entire sequence of calculations, comprised of the initial period of substrate growth followed by a period of substrate oxidation. With the primary focus on factors affecting oxidation, the present simulations all started with the same set of 100 grown substrates. 


\section{Results and discussion}

KMC simulations were performed at 1500, 2000, and $2500 \mathrm{~K}$. In each simulation, the graphene structure was first grown from an initial coronene substrate for $5 \mathrm{~ms}$. The temperature was held constant in each simulation run. The pressure was held constant at 1 atm. The gas-phase composition was also held constant with mole fractions $x_{\mathrm{C}_{2} \mathrm{H}_{2}}=x_{\mathrm{H}_{2}}=0.1$ and $x_{\mathrm{H}}=0.01$, representative of soot-forming flame environments. After $5 \mathrm{~ms}$ of growth at these conditions, $\mathrm{O}_{2}$ was added to the gaseous environment and acetylene was removed (i.e., $x_{\mathrm{C}_{2} \mathrm{H}_{2}}$ was set to 0 ) to simulate a regime that we refer to as pure oxidation [40].

\subsection{Principal oxidation pathways}

First, we re-examined the major pathways of the oxidation system. As mentioned briefly in Section 2, the simulations performed in the previous study [40] were all carried out starting from the initial substrate, coronene; i.e., each individual run started with a period of $5 \mathrm{~ms}$ growth of coronene followed by a period of $2 \mathrm{~ms}$ oxidation of the grown graphene structure. With the present focus on isolating factors affecting oxidation (and not growth), we repeated these simulations, but now keeping the grown graphene structure the same for all simulations of oxidation. For each case, this grown graphene structure was obtained in a series of only-growth simulations at the case conditions, saved, and used as the starting substrate for all the oxidation runs of the case. The results of these repeated simulation runs were principally the same as the previous ones. We report some of these results here for completeness of and in support of the discussion that follows.

The computed time evolution of the substrate size and five-member ring fraction for a range of oxygen concentrations are depicted in Fig. 1 for each temperature. The substrate size, plotted in the left-hand panels of Fig. 1, initially increases, during the growth period, and then decreases 
when $\mathrm{O}_{2}$ is added to and $\mathrm{C}_{2} \mathrm{H}_{2}$ is removed from the gas phase. The extent of the decrease in the substrate size is intensified with the rise in temperature and in $\mathrm{O}_{2}$ concentration. Also of note is a slight decline in the substrate size when $\mathrm{C}_{2} \mathrm{H}_{2}$ is removed but no $\mathrm{O}_{2}$ is added to the gas phase, especially evident at $1500 \mathrm{~K}$. This decline is caused by the thermal decomposition of reaction intermediates that are present on the graphene edge at the time of the abrupt secession of the growth. We account for this phenomena when we compare simulations to experiment by introducing an annealing period between growth and oxidation, discussed further in Section 3.4.
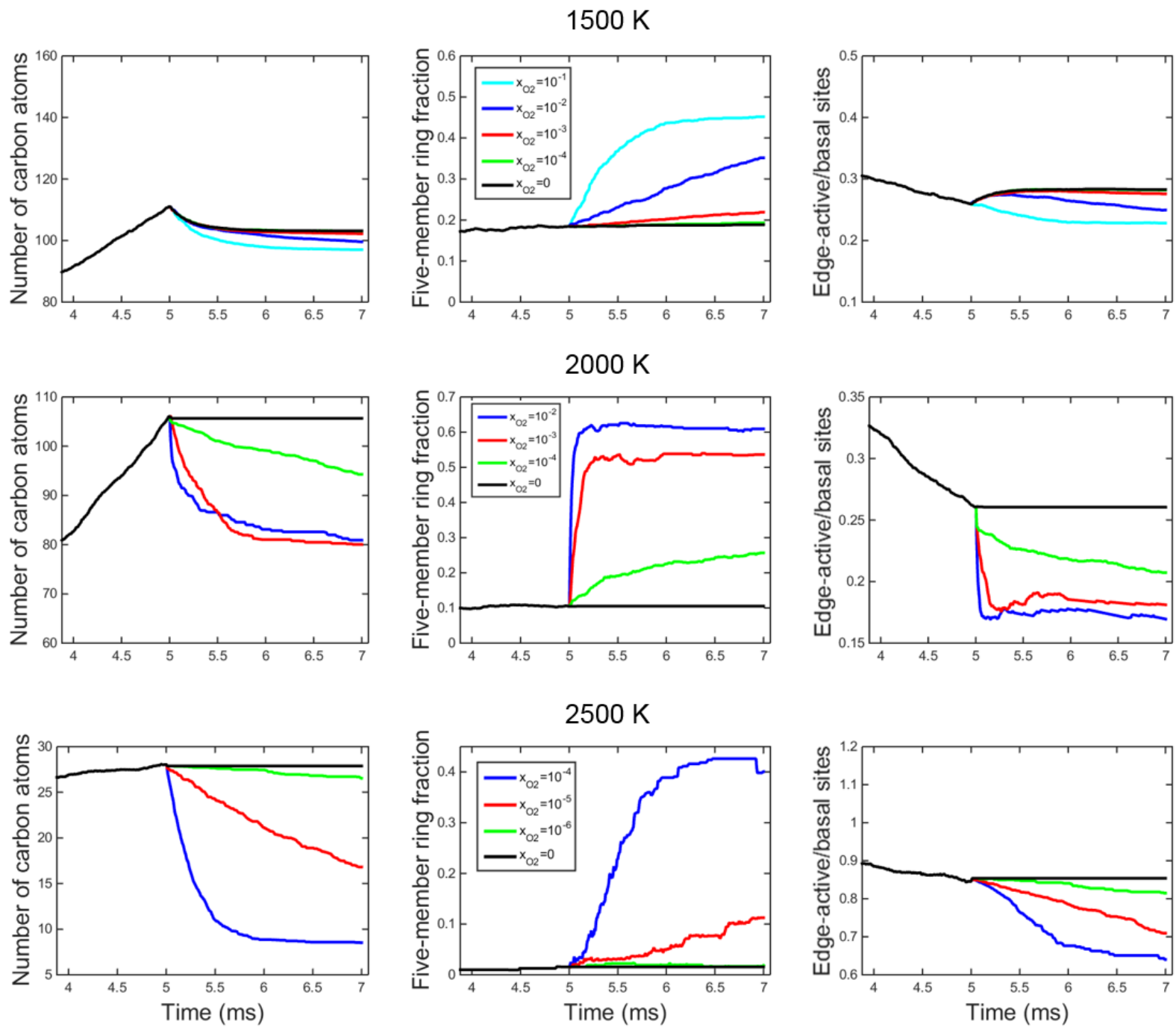

Fig. 1 - Substrate size (left), five-member ring fraction (center), and $\mathrm{C}_{\text {edge }}-\mathrm{H} / \mathrm{C}_{\text {basal }}$ site ratio (right) 
The five-member ring fractions, depicted in the center panels of Fig. 1, reveal that the addition of $\mathrm{O}_{2}$ promotes the formation of five-member rings embedded in the graphene sheet. The increase in the five-member rings is primarily due to their formation via thermal decomposition of oxyradicals
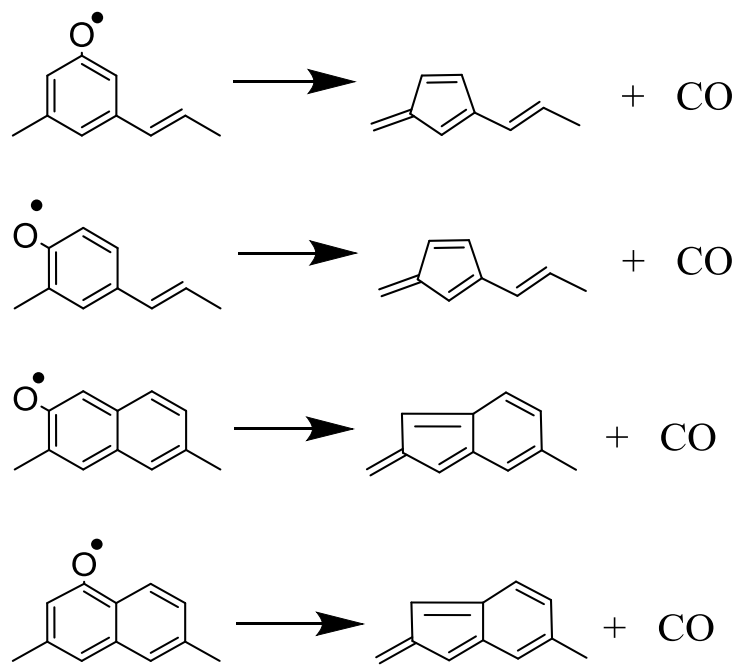

where the reaction numbers are those of Table S1 of the Supplementary Data. The reaction mechanism constrains two five-member rings from migrating next to each other, so if too many five-member rings form in close proximity to each other, they cannot migrate across the graphene edge and thermally desorb.

The KMC results revealed two major, competing pathways for an oxyradical originating in $\mathrm{O}_{2}$ reaction with a surface radical, displayed schematically in Fig. 2. In the first pathway, the oxyradical undergoes thermal decomposition to form a five-member ring and expel $\mathrm{CO}$ - the actual oxidation step. In the second one, a gas phase hydrogen atom adds to the oxyradical forming $\mathrm{OH}$, to which another hydrogen atom adds to expel $\mathrm{H}_{2} \mathrm{O}$ and regenerate a new aromatic radical site - a pathway recycling the aromatic-radical site. There are several other reactions in the mechanism that accomplish the edge-radical recycling. The competition between the two 
pathways remains the same as was quantified in the previous study [40]. At lower temperatures, the regeneration pathway is dominant over the thermal decomposition pathway. At high temperatures, the oxidation pathway is dominant. This switch is a result of thermal decomposition being a higher activation-energy process than regeneration.

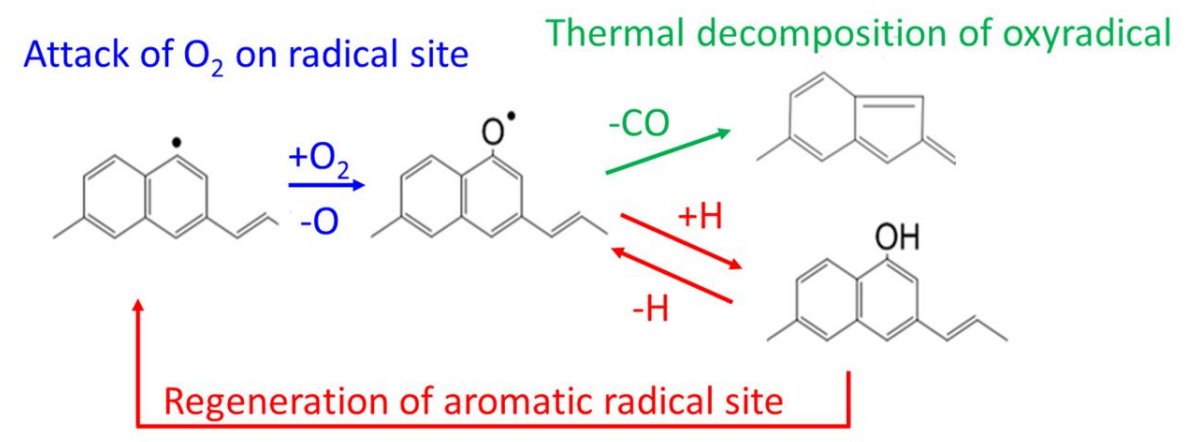

Fig. 2 - Diagram of the two major pathways: oxidation (green) and regeneration of an aromatic radical site (red) [40].

\subsection{Effect of regeneration on the rate of oxidation}

While the relative rates of the two pathways switch dramatically in the temperature range studied (cf. Fig. S1 in the Supplementary Data), the influence of the regenerative pathway on the overall, bulk oxidation rate is not very large. Indeed, let us examine the results shown in Table 1 that reports the bulk oxidation rates obtained in additional KMC runs, performed at the same conditions as those in Section 3.1 but with regeneration pathways excluded from the simulations.

Table 1 - Oxidation rates $(\mathrm{C}$-atom/ms) computed with and without regeneration pathway included.

\begin{tabular}{|c|c|c|c|c|c|c|c|c|c|}
\hline \multicolumn{2}{|c|}{ Temperature (K) } & \multicolumn{2}{|l|}{1500} & \multicolumn{3}{|c|}{2000} & \multicolumn{3}{|c|}{2500} \\
\hline$x_{\mathrm{O}_{2}}$ & $10^{-3}$ & $10^{-2}$ & $10^{-1}$ & $10^{-4}$ & $10^{-3}$ & $10^{-2}$ & $10^{-6}$ & $10^{-5}$ & $10^{-4}$ \\
\hline With & 24.5 & 26.6 & 36.4 & 15.3 & 45.0 & 54.1 & 7.0 & 10.2 & 49.7 \\
\hline Without & 27.2 & 29.8 & 36.4 & 18.6 & 53.8 & 54.1 & 7.0 & 10.2 & 49.7 \\
\hline
\end{tabular}


At $1500 \mathrm{~K}$, the initial oxidation rates increase by up to $12 \%$ when regeneration was excluded. In spite of the dominant pathway for oxyradicals at $1500 \mathrm{~K}$ being the regeneration pathway, excluding the regeneration reactions leads only to a marginal increase in the frequency of oxidation of six- and five-member rings and hence only a marginal increase in the oxidation rates. In other words, at these conditions the fast regeneration reactions simply recycle the aromatic oxyradicals. The results at $2000 \mathrm{~K}$ are similar to those at $1500 \mathrm{~K}$ with the oxidation rates increasing by up to $21 \%$ for the simulations without regeneration. At $2500 \mathrm{~K}$, there is no change in the oxidation rates when regeneration reactions are excluded, because the oxidation pathway is already completely dominant over the regeneration pathway at this high temperature.

The fact that regeneration does not affect greatly the bulk oxidation rate may help in developing reduced models of soot oxidation at flame conditions and, to some extent, may justify the use of the one-step model (1) in combustion simulations.

\subsection{Reactivity of graphene edges with embedded five-member rings}

The results of Section 3.1 indicate that accumulation of five-member rings at the graphene edge leads to its reduced reactivity. This is manifested by the decaying rates of graphene-edge oxidation. Indeed, as can be seen in Fig. 1, the profiles of the substrate size during the oxidation are non-linear in time, implying time-varying — decaying — rates of oxidation. Since the gaseous environment is maintained unchanged during an individual simulation, the only varying property affecting the rate of oxidation is the edge density of reactive sites and their reactivity towards oxidation.

The notion that incorporation of five-member rings into graphene edges makes it less reactive is counter intuitive at first, as five-member rings are usually considered to be less stable $[15,29,57]$. To explain the computed behavior, we need to consider the morphology of the 
graphene edge. Fundamentally, a graphene edge can be in two forms, armchair and zigzag. At combustion conditions we presume that all edge sites are saturated with $\mathrm{H}$ atoms and reaction is initiated by abstracting an $\mathrm{H}$ atom forming a surface radical $[5,18,19,58]$. Left to growth only, a finite-size armchair edge evolves quickly into zigzag edges [23]. Zigzag edges can also grow, rate-limited by surface nucleation occurring either at edge corners or though chemisorbed and migrating five-member rings [29], both slower than the growth of armchair edges (via the HACA mechanism $[5,58]$, for example).

Oxidation is essentially controlled by decomposition of surface oxyradicals. A six-memberring oxyradical decomposes by expelling $\mathrm{CO}$ and forming a five-member ring [59]. Recent theoretical studies of elementary reactions concluded that inner zigzag oxyradical sites decompose much slower, if at all, than corner zigzag and armchair sites at combustion conditions $[34,36]$. These results imply that armchair-edge oxidation should proceed as random removal of individual edge sites while that of zigzag edges as a zipper-type reaction process. Literature is rather conflicting on comparison of armchair and zigzag oxidation reactivity. For instance, the early microscopy work of Thomas and Hughes [60] reported slightly higher recession rates of zigzag than armchair edges, yet a recent review concludes that armchair carbon atoms are more reactive than zigzag ones [61]. We note that the close proximity in numerical measures of reactivity (those observed by Thomas and Hughes) for the two edges could be consistent with the theoretical predictions that the oxidation (and growth [44]) rates of armchair and corner zigzag sites are close to each other, and the difference in experimentally observed edge evolution is due to cooperative phenomena of elementary reaction steps and edge geometry.

Returning to the present simulations, graphene edges formed in the growth period are both zigzag and armchair, as illustrated in Fig. 3a. When the oxidation period begins, the zigzag sites 
remain largely intact and the armchair sites convert into five-member rings, as can be seen by comparison between the edge fragment enclosed in the green box in Fig. 3a with the corresponding red-marked edge fragment in Fig. 3b.

(a) $4.8 \mathrm{~ms}$

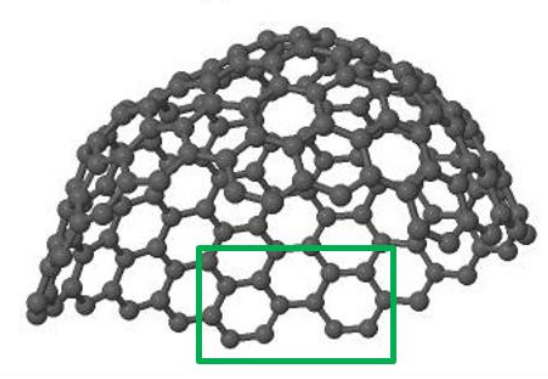

(b) $6.6 \mathrm{~ms}$

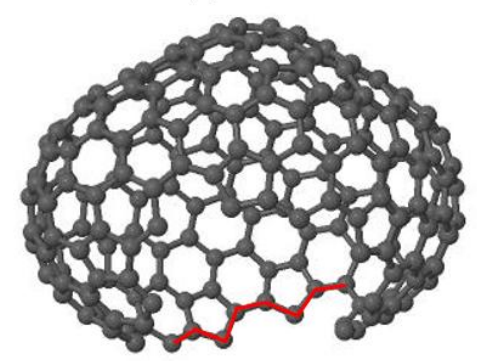

Fig. 3 - Graphene structures obtained in a KMC simulation at $2000 \mathrm{~K}$ and $x_{\mathrm{O}_{2}}=10^{-3}$ (a) toward the end of the growth period, just before the onset of oxidation, and (b) after $1.6 \mathrm{~ms}$ of oxidation. The $\mathrm{H}$ atoms saturating the edge carbon atoms are not shown for clarity.

While the emerged red-marked fragment with embedded five-member rings is not a zigzag edge fragment, it is also not an armchair. Analysis of oxidation pathways for such embedded five-member rings

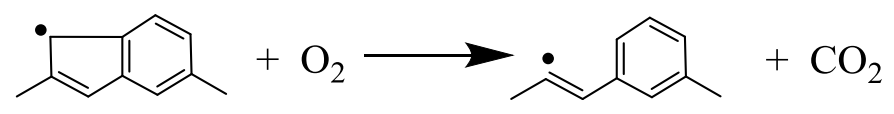

yielded rates substantially higher than those of inner zigzag sites [34] but much (over four orders of magnitude) lower than those for six-member rings of an armchair edge [15, 40].

The phenomenon of decreasing oxidative reactivity of soot over time has been observed experimentally. In a study by Vander Wal and co-workers [12], soot samples were collected from diesel particulate filters after different kinds of engine testing, and the nanostructure of primary particles within the aggregates was observed using HRTEM imaging. They found that 
samples with longer exposure to oxidation conditions had a higher fraction of densified particles than samples collected over limited-time low-load conditions. The authors [12] explained that the densification occurred because oxidation preferentially removed amorphous carbon relative to graphitic carbon causing a decrease in the number of reactive edge-site carbon atoms relative to the number of more stable basal-plane carbon atoms and, hence, a decrease in oxidative reactivity. Similar observations were reported by Jaramillo et al. [16] and Fang and Lance [62].

In the present KMC simulations, we observed a similar decrease in the ratio of reactive edge sites to basal-plane carbon atoms during oxidation, as evidenced by the computed ratios depicted in the right-hand panels of Fig. 1. At all temperatures the ratio is decreasing during the growth period of the simulation. This is readily understood: carbon addition reactions reproduce edge sites while incorporating the carbon atoms of the preceding reactive sites into the basal plane. One would expect a reverse outcome in oxidation: removal of peripheral edge carbons would leave behind reactive sites while decreasing the number of inner, basal-plane carbons. However, the simulations showed that the $\mathrm{C}_{\text {edge }}-\mathrm{H} / \mathrm{C}_{\text {basal }}$ site ratio is decreased during oxidation. The results at $1500 \mathrm{~K}$, displaying an initial increase in this site ratio, may seem contradictory to this assessment. A closer examination, adding an annealing period between growth an oxidation (see Section 3.4), revealed that the rise in the site ratio is due to thermal desorption of carbon, but the oxidation keeps decreasing it, as demonstrated in Fig. 4. The reason for the site ratio decrease, as explained above, is the formation of five-member rings that accumulate and create lessreactive zigzag edges. These considerations lead us to conclude that the observed decrease in reactivity over time seen in our results is in accord with the phenomena observed in experiment $[12,16,62]$. 


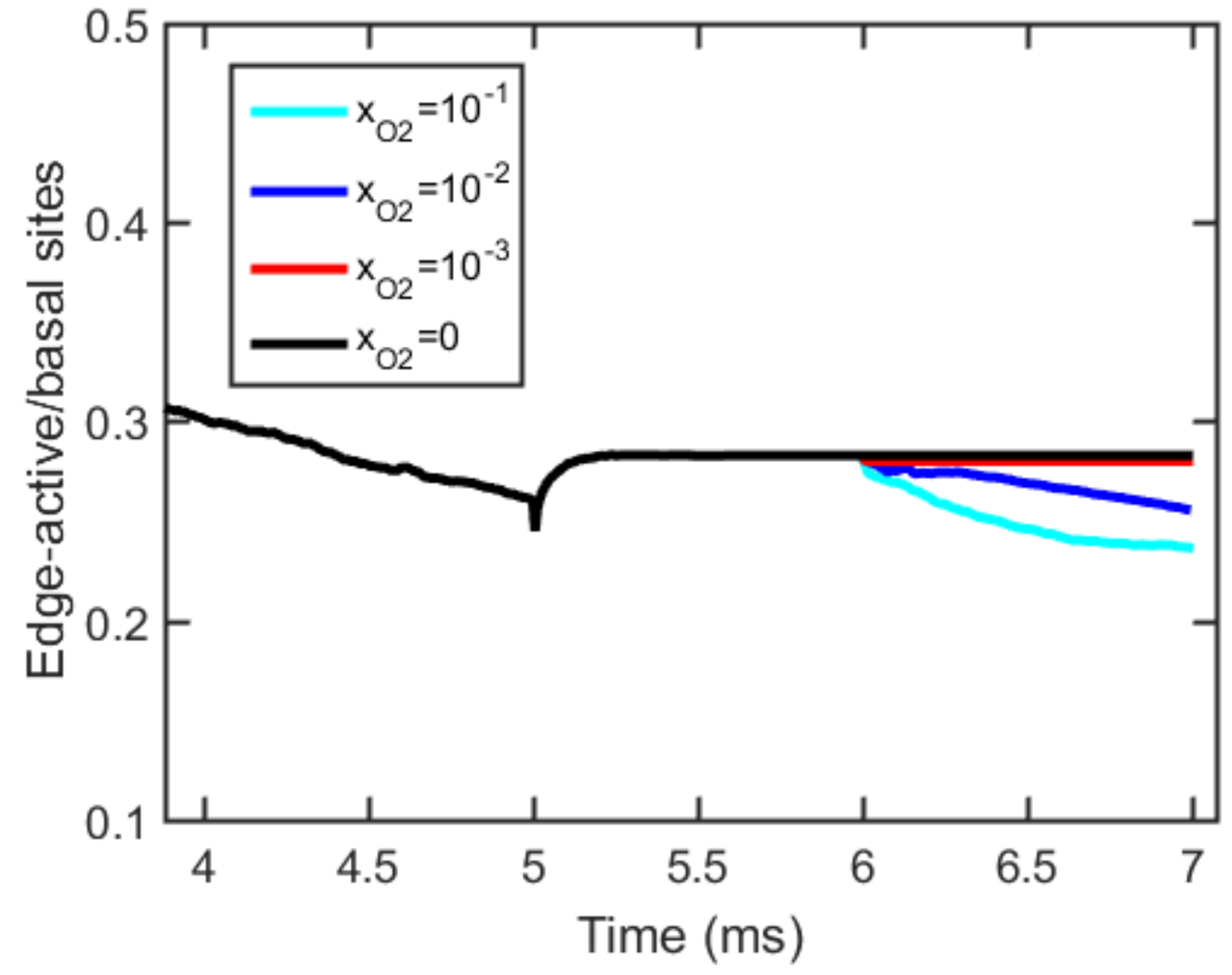

Fig. 4. $-\mathrm{C}_{\text {edge }}-\mathrm{H} / \mathrm{C}_{\text {basal }}$ site ratio computed at the same conditions as those of upper left corner of Fig. 1 with the addition of $1 \mathrm{~ms}$ annealing period between growth and oxidation.

\subsection{Effect of graphene curvature on the rate of oxidation}

In an earlier experimental study, Vander Wal and Tomasek [11] investigated the dependence of oxidation rates on particle nanostructure. Soot was synthesized using three different fuels (acetylene, benzene, and ethanol) to achieve different nanostructures. Burnout rates were measured for the initial stage of oxidation, when less than $25 \%$ of the initial mass was lost. The authors [11] found that the burnout rates for ethanol- and benzene-derived soot were higher than those for acetylene-derived soot by nearly a factor of five. Fringe lattice analysis revealed that the fringe length distribution of benzene-derived soot indicated shorter, unaligned graphene 
segments, which is indicative of a high ratio of edge-site carbon atoms to basal-plane carbon atoms and, therefore, higher reactivity. However, ethanol-derived soot had longer graphene segments, like acetylene-derived soot but still had much higher burnout rates. Examination of the HRTEM images showed that ethanol-derived soot had much greater curvature than acetylene-derived soot. Vander Wal and Tomasek conjectured that a higher degree of curvature increases the imposed bond strain between C-C bonds thereby decreasing their resistance toward oxidation and thus explained why the ethanol-derived soot had higher burnout rates than the acetylene-derived soot despite having a similar fringe length distribution.

Our numerical prediction that incorporation of five-member rings into graphene edges causes the decay in the oxidation rate could be seemingly perceived as contradictory to the experimentally derived conclusion of Vander Wal and Tomasek [11] that graphene curvature increases the oxidation rate of soot. Yet, further analysis revealed not only that there is no controversy but identified an additional feature of the graphene oxidation.

This next set of tests was performed by starting oxidation with graphene structures of differing curvature. Such two different nanostructures were obtained in KMC simulations of graphene growth at two different temperatures, 1500 and $2000 \mathrm{~K}$. The degree of curvature was quantified by the five-member ring fraction, $f_{\mathrm{R} 5}$. The substrates grown at $1500 \mathrm{~K}$ had an average $f_{\mathrm{R} 5}$ of 0.18 , while those grown at $2000 \mathrm{~K}$ had an average $f_{\mathrm{R} 5}$ of 0.10 , thus indicating a higher degree of curvature for the substrates grown at $1500 \mathrm{~K}$. Fig. 5 illustrates the differences in curvature by showing representative structures after $5 \mathrm{~ms}$ of growth at each temperature. 
(a) $1500 \mathrm{~K}$

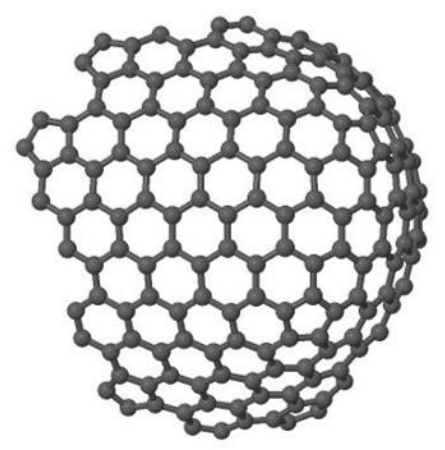

(b) $2000 \mathrm{~K}$

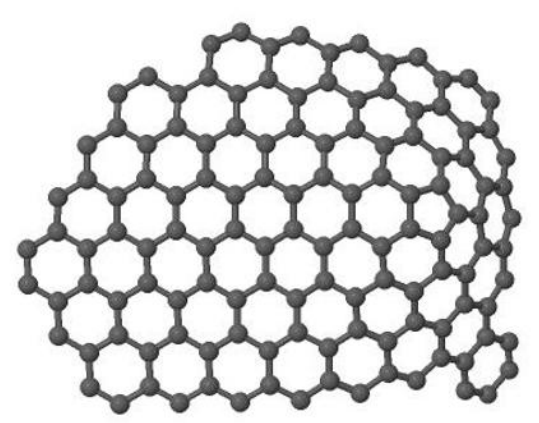

Fig. 5 - Snapshots of substrates grown at (a) $1500 \mathrm{~K}$ and (b) $2000 \mathrm{~K}$ at $5 \mathrm{~ms}$. The $\mathrm{H}$ atoms saturating the edge carbon atoms are not shown for clarity.

The two graphene structures were subjected to oxidation at different oxygen concentrations, each substrate at three different temperatures: 1500, 2000, and $2500 \mathrm{~K}$. The KMC simulations were now performed in three stages: growth, annealing, and oxidation. Like in prior simulations, the graphene sheet was grown for $5 \mathrm{~ms}$ starting with an initial coronene substrate. After that, the grown graphene structure underwent an annealing period for $1 \mathrm{~ms}$, during which $\mathrm{C}_{2} \mathrm{H}_{2}$ and $\mathrm{H}$ were removed from the gaseous environment and $\mathrm{O}_{2}$ was not yet added. The purpose of the annealing period was to allow for radical sites that existed at $5 \mathrm{~ms}$ to thermally decompose. The reason for the introduction of this period in our study was to mimic physical processes possibly occurring in experimental setups during transferring soot particles from the growth environment to the oxidation one [11]. This should not be confused with the concept of "thermal annealing" as it is often termed in the carbon literature $[63,64]$. The latter is probably more comparable with our oxidation period, discussed next, when all processes occur simultaneously: we study the dynamics of graphene-edge evolution by allowing all feasible elementary reactions proceed at the same time. In other words, there is no annealing per se, but it is a competition between thermal desorption reactions and oxidative attacks, the phenomena we examine next. 
At $6 \mathrm{~ms}$, molecular oxygen and atomic hydrogen were added to the gaseous environment for $2 \mathrm{~ms}$ of oxidation. The temperature and pressure remained unchanged throughout the simulation. To stay closer to the experimental procedure of Vander Wal and Tomasek [11], we examined here the initial rates of oxidation. The obtained results are reported in Table 2. (We note here that the experimental results of Vander Wal and Tomasek [11] were carried out close to $1000 \mathrm{~K}$, well below $1500 \mathrm{~K}$, the intended lower bound of the temperature range for our mechanism. As a test, we performed simulations at $1000 \mathrm{~K}$ as well as at a lower $\mathrm{H}$-atom concentration, $x_{\mathrm{H}}=1 \times 10^{-3}$ [Tables S2 and S3 of the Supplementary Data]; and the results obtained were similar, qualitatively, to those reported in Table 2.)

Table 2 - Initial oxidation rates (C-atom/ms) for substrates with different curvature.

\begin{tabular}{|c|c|c|c|c|c|c|c|c|c|c|}
\hline \multicolumn{2}{|c|}{ Oxidation $T(\mathrm{~K})$} & \multicolumn{3}{|c|}{1500} & \multicolumn{3}{|c|}{2000} & \multicolumn{3}{|c|}{2500} \\
\hline & $x_{\mathrm{O}_{2}}$ & $10^{-3}$ & $10^{-2}$ & $10^{-1}$ & $10^{-4}$ & $10^{-3}$ & $10^{-2}$ & $10^{-6}$ & $10^{-5}$ & $10^{-4}$ \\
\hline $\begin{array}{c}\text { Growth } \\
T(\mathrm{~K})\end{array}$ & $f_{\mathrm{R} 5}$ & & & & & & & & & \\
\hline 1500 & 0.18 & 24.5 & 26.6 & 36.4 & 48.8 & 77.6 & 82.1 & 42.2 & 44.3 & 59.7 \\
\hline 2000 & 0.10 & 6.4 & 9.1 & 21.2 & 15.3 & 45.0 & 54.1 & 9.3 & 10.7 & 29.0 \\
\hline
\end{tabular}

Inspection of the results reported in Table 2 indicates that in all nine cases tested the oxidation rates were higher, by a factor of 1.5 to 4.6 , for graphene with larger starting curvature. This comparison shows that our KMC model does reproduce the phenomenon seen in the experiments of Vander Wal and Tomasek [11]. Then how can it be that the same mechanistic feature - incorporation of five-member rings into a growing edge of graphene - explains both the decay of the oxidation rate in time and the faster initial oxidation of a more curved graphene? 
In search for answers we turn to analysis of the underlying reaction statistics. In the KMC model, in addition to carbon being removed from the graphene edge by oxidation, reactions (R51)-(R54) and (R90), it is also eliminated by thermal desorption
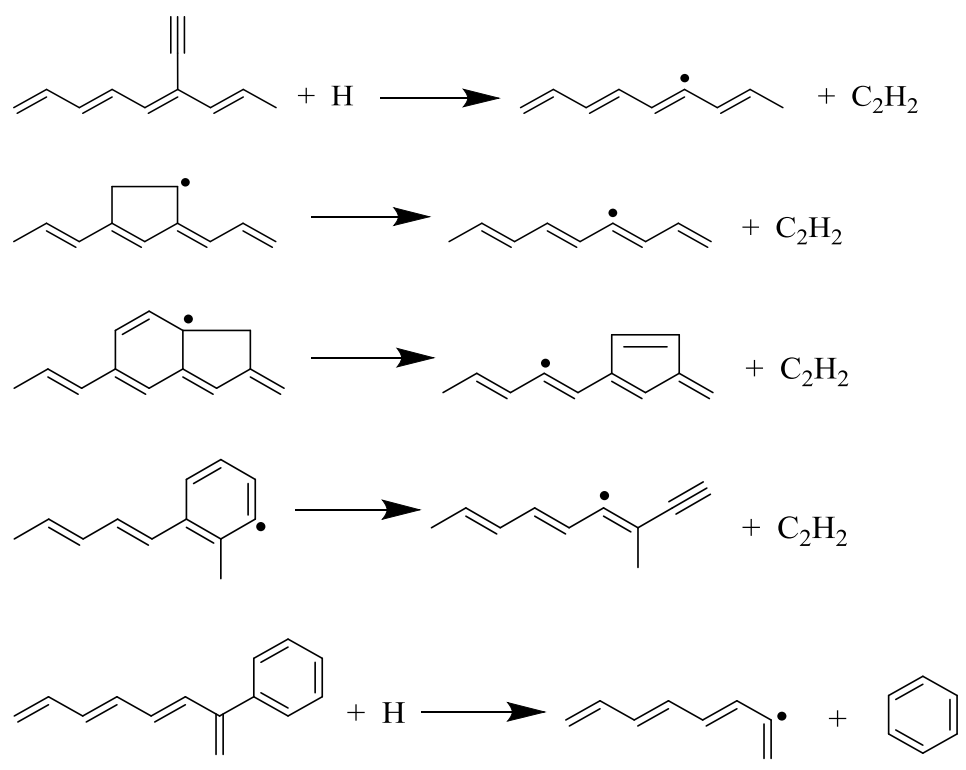

In these simulation runs, the frequency of oxidation reactions (R51)-(R54) was approximately the same for substrates grown at 1500 and $2000 \mathrm{~K}$. However, the thermal desorption reactions, primarily (R5) and (R7), occurred between two to four times as often for the substrates grown at $1500 \mathrm{~K}$ as for those grown at $2000 \mathrm{~K}$ during the first $0.2 \mathrm{~ms}$ of oxidation (Fig. 6). Therefore, it is the thermal desorption that accounts for the difference in the oxidation rates.

Further examination of the data displayed in Fig. 6 shows that the difference in frequency of thermal desorption reactions between the two sets of substrates is much smaller after the initial $0.2 \mathrm{~ms}$ of oxidation for all simulation conditions. This suggests that the difference in oxidation rates between substrates with different curvature occurs primarily at the beginning of oxidation and happens because substrates with higher curvature form a larger number of sites that can potentially desorb. 

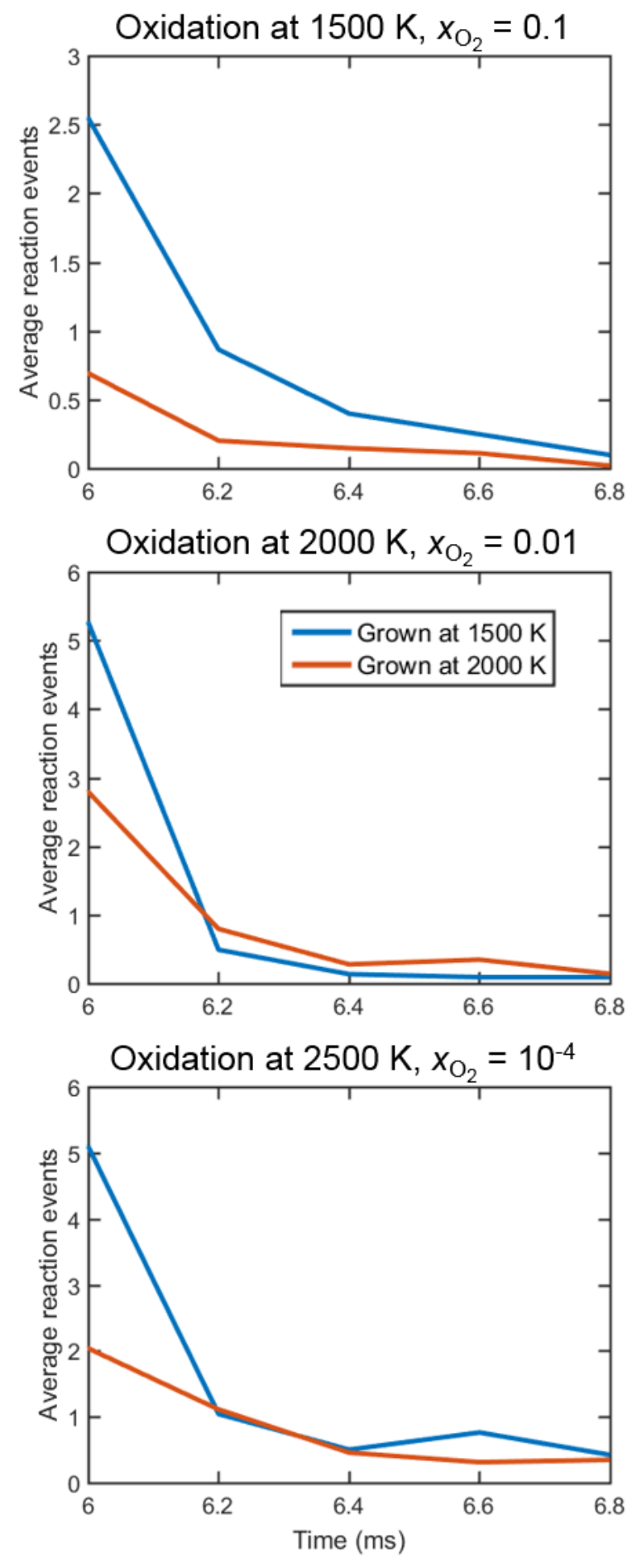

Fig. 6 - Event count for thermal desorption reactions versus time for substrates oxidized at three sets of conditions. 
The reaction count statistics indicate that the metric of curvature is more complex than simply knowing the fraction of five-member rings in the graphene sheet. Five-member rings can affect oxidation differently, depending on their location in the graphene sheet, i.e., if they are on an edge or embedded. The time evolution of the different types of five-member rings is plotted in Fig. 7. Inspection of these results indicates that at the beginning of oxidation substrates grown at $1500 \mathrm{~K}$ have a higher number of free five-member rings (red lines in Fig. 7) that can be thermally desorbed compared to substrates grown at $2000 \mathrm{~K}$, thus explaining the difference in the reaction counts presented earlier. As oxidation progresses, the free five-member rings are thermally desorbed and their number decreases close to zero for the substrates grown at both temperatures. The number of five-member rings at armchair sites (blue lines) increases for both substrates due to thermal decomposition of oxyradicals to form five-member rings. These latter five-member rings form zigzag edges that lead to a decrease in oxidative reactivity. The number of bay-capped five-member rings (black lines) decrease only slightly for the substrates at both temperatures and do not seem to affect the oxidation rate.

Thus, while the total number of five-member rings increases during oxidation for the substrates grown at 1500 and $2000 \mathrm{~K}$, their individual histories vary and have different effects on the oxidation rate. Analysis of the graphene-edge site distribution over time further supports this conclusion. Figure 8 displays population of pertinent edge sites at several instances of reaction; the sites are identified in Fig. 9. Inspection of these results indicates that the fraction of freeedge sites decreases during the oxidation stage for both substrates. The substrates grown at 1500 $\mathrm{K}$ have a significant fraction of free-edge- 5 sites, five-member rings that thermally desorb, which is evidenced by a substantial decrease in these sites during the annealing period. By contrast, such five-member rings barely appear on substrates grown at $2000 \mathrm{~K}$. This discrepancy in free- 
edge-5 sites further illuminates that a higher oxidation rate of more curved graphene is due to the presence of five-member rings that can thermally desorb. At the same time, the total number of zigzag sites, the sum of zigzag and zigzag-5 sites, increases as oxidation progresses at both temperatures. Accumulation of such less-reactive-to-oxidation sites causes the decrease in the oxidation rate over time. These distinct aspects of graphene edge morphology reconcile the two seemingly contradictory conclusions about curvature effects on oxidation rates.
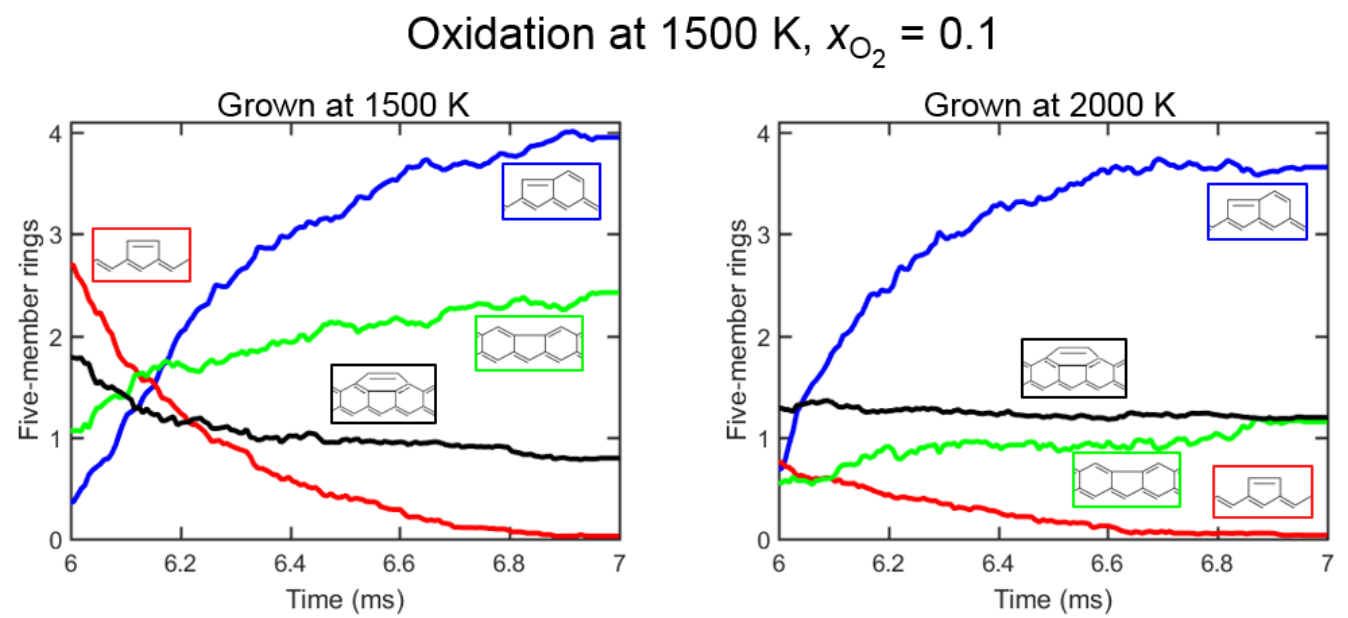

Fig. 7 - Number of five-member rings versus time for substrates grown at 1500 (left) or $2000 \mathrm{~K}$ (right), and oxidized at $1500 \mathrm{~K}$ with $x_{\mathrm{O}_{2}}=0.1$. 

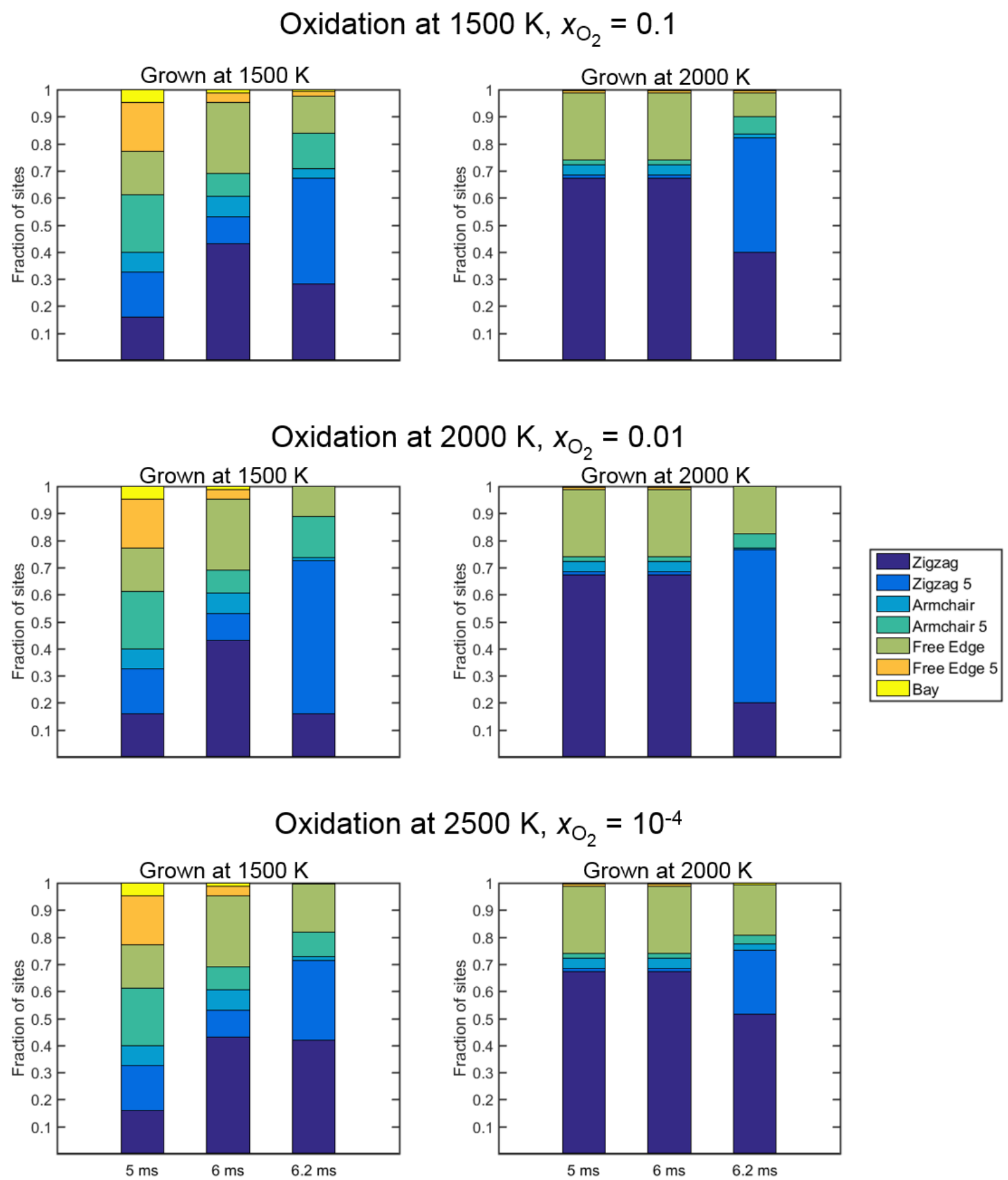

Fig. 8 - Site distribution at $5 \mathrm{~ms}$ (start of annealing), $6 \mathrm{~ms}$ (start of oxidation), and $6.2 \mathrm{~ms}$ (after $0.2 \mathrm{~ms}$ of oxidation) for substrates grown and oxidized under different conditions. 


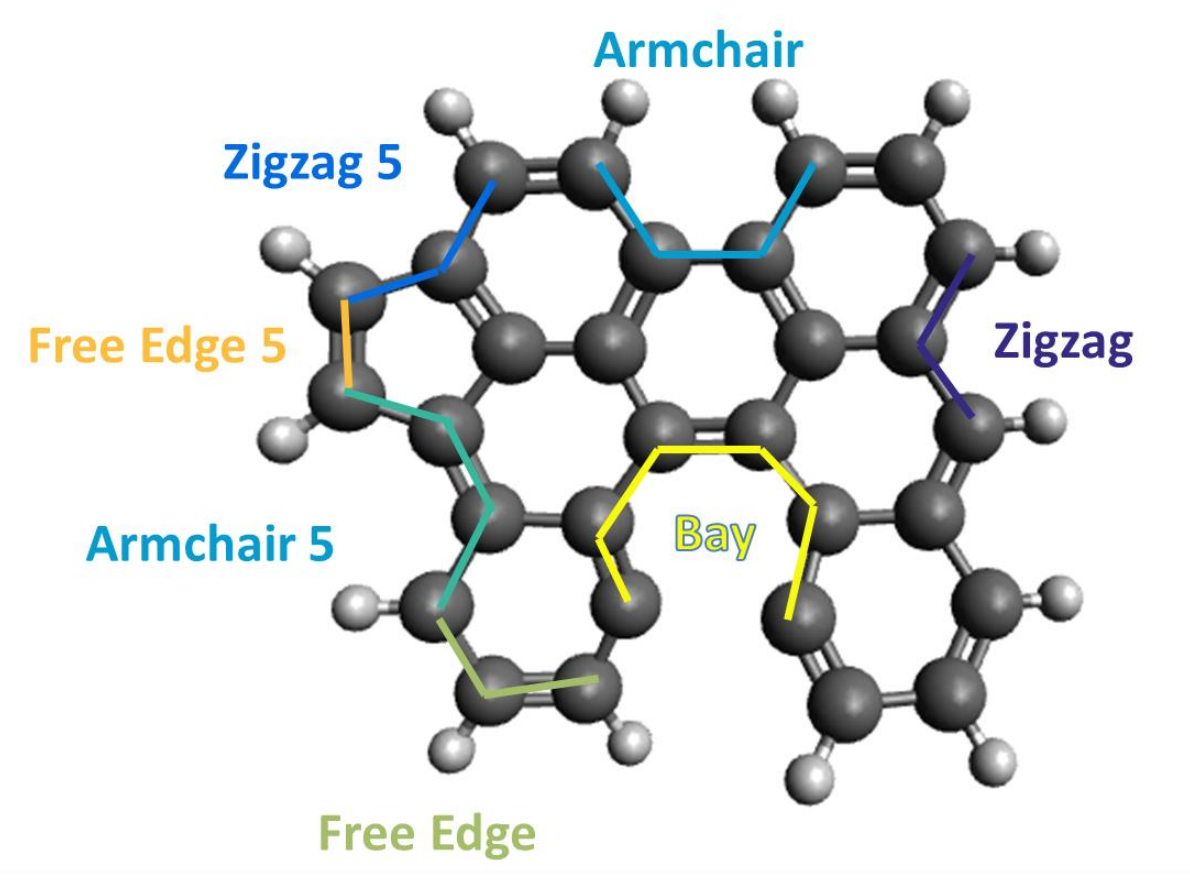

Fig. 9 - Diagram of sites used in Fig. 8.

\section{Conclusions}

Kinetic Monte-Carlo simulations of the graphene-edge evolution in combustion-like environments revealed that oxidation by molecular oxygen exhibits two principal pathways: thermal decomposition of oxyradicals and regeneration of aromatic radical sites. Their competition is temperature dependent, with former dominating at higher and the latter at lower temperatures. Yet, the overall oxidation rate at the conditions tested is not substantially affected by presence or absence of the regeneration pathway. This may help in developing reduced models of soot oxidation at flame conditions and, to some extent, may justify the use of the onestep model (1) in combustion simulations.

The overall oxidation rate of the graphene substrate was computed to be time dependent, with reactivity decreasing over time as the ratio of reactive edge sites decreases relative to the number of basal-plane carbon atoms. At the same time, the oxidation rate was found to be higher for graphene with a higher initial curvature. Both results are in accord with experimental 
observations [11, 12]. Analysis showed that distinct aspects of graphene-edge morphology are responsible for curvature either raising or reducing the oxidative reactivity of the graphene edge.

\section{Acknowledgements}

The authors acknowledge financial support by the US Army Corps of Engineers, Humphreys Engineering Center Support Activity, under Contract No. W912HQ-07-C-0044. This research used computational resources of the National Energy Research Scientific Computing Center, which is supported by the Office of Science of the US Department of Energy under Contract No. DE- AC02-05CH11231.

\section{References}

[1] Boudart M, Djéga-Mariadassou G. Kinetics of Heterogeneous Catalytic Reactions. Princeton, N.J.: Princeton University Press; 1984.

[2] Steinfeld JI, Francisco JS, Hase WL. Chemical Kinetics and Dynamics. Upper Saddle River, N. J.: Prentice Hall; 1999.

[3] Carmer CS, Weiner B, Frenklach M. Molecular dynamics with combined quantum and empirical potentials: C2H2 adsorption on Si(100). J Chem Phys. 1993;99:1356-72.

[4] Harris J, Kasemo B. On precursor mechanisms for surface reactions. Surf Sci. 1981;105:L281-L7.

[5] Frenklach M. Reaction mechanism of soot formation in flames. Phys Chem Chem Phys. 2002;4(11):2028-37.

[6] Bockhorn H, D'Anna A, Sarofim AF, Wang H. Combustion Generated Fine Carbonaceous Particles. Karlsruhe, Germany: KIT Scientific; 2007.

[7] Wang H. Formation of Nascent Soot and Other Condensed-phase Materials in Flames. Proc Combust Inst. 2011;33:41-67.

[8] Balthasar M, Mauss F, Knobel A, Kraft M. Detailed modeling of soot formation in a partially stirred plug flow reactor. Combust Flame. 2002;128:395-409.

[9] Dato A, Frenklach M. Substrate-free microwave synthesis of graphene: experimental conditions and hydrocarbon precursors. New J Phys. 2010;12:125013.

[10] Stanmore BR, Brilhac JF, Gilot P. The oxidation of soot: a review of experiments, mechanisms and models. Carbon. 2001;39:2247-68.

[11] Vander Wal R, Tomasek AJ. Soot oxidation: Dependence upon initial nanostructure. Combust Flame. 2003;134:1-9.

[12] Vander Wal R, Yezerets A, Currier NW, Kim DH, Wang CM. HRTEM study of diesel soot collected from diesel particulate filters. Carbon. 2007;45:70-7.

[13] Ma X, Zangmeister CD, Zachariah MR. Soot oxidation kinetics: A comparison study of two tandem ionmobility methods. J Phys Chem C. 2013;117:10723-9.

[14] Radovic LR. Active sites in graphene and the mechanism of $\mathrm{CO}_{2}$ formation in carbon oxidation. $\mathrm{J}$ Am Chem Soc. 2009;131:17166-75.

[15] Raj A, Yang SY, Cha D, Tayouo R, Chung SH. Structural effects on the oxidation of soot particles by $\mathrm{O}_{2}$ : Experimental and theoretical study. Combust Flame. 2013;160:1812-26.

[16] Jaramillo IC, Gaddam CK, Vander Wal RL, Lighty JS. Effect of nanostructure, oxidative pressure and extent of oxidation on model carbon reactivity. Combust Flame. 2015;162:1848-56.

[17] Nagle J, Strickland-Constable RF. Oxidation of carbon between 1000-2000 ${ }^{\circ} \mathrm{C}$. Proceedings of the Fifth Carbon Conference. Oxford: Pergamon 1962, p. 154-64.

[18] Frenklach M. A unifying picture of gas phase formation and growth of PAH, soot, diamond and graphite. In: Tarter JC, Chang S, DeFrees DJ, eds. Carbon in the Galaxy: Studies From Earth and Space: NASA Conference Publication 3061 1990, p. 259-73.

[19] Frenklach M, Wang H. Detailed modeling of soot particle nucleation and growth. Proc Combust Inst. 1991;23:1559-66. 
[20] $\mathrm{Yu}$ T, Lin MC. Kinetics of the $\mathrm{C}_{6} \mathrm{H}_{5}+\mathrm{O}_{2}$ reaction at low temperatures. J Am Chem Soc 1994;116:9571-6.

[21] Frenklach M, Wang H. Detailed mechanism and modeling of soot particle formation. In: Bockhorn H, ed. Soot Formation in Combustion: Mechanisms and Models. Heidelberg: Springer-Verlag 1994, p. 165-92.

[22] Appel J, Bockhorn H, Frenklach M. Kinetic modeling of soot formation with detailed chemistry and physics: Laminar premixed flames of $\mathrm{C}_{2}$ hydrocarbons. Combust Flame. 2000;121:122-36.

[23] Frenklach M, Schuetz CA, Ping J. Migration mechanism of aromatic-edge growth. Proc Combust Inst. 2005;30:1389-96.

[24] Violi A. Cyclodehydrogenation reactions to cyclopentafused polycyclic aromatic hydrocarbons. J Phys Chem A. 2005;109(34):7781-7.

[25] Tokmakov IV, Kim G-S, Kislov VV, Mebel AM, Lin MC. The reaction of phenyl radical with molecular oxygen: A G2M study of the potential energy surface. J Phys Chem A. 2005;109(27):6114-27.

[26] Whitesides R, Domin D, Salomón-Ferrer R, Lester Jr WA, Frenklach M. Embedded-ring migration on graphene zigzag edge. Proc Combust Inst. 2009;32:577-83.

[27] Whitesides R, Domin D, Salomón-Ferrer R, Lester WA, Jr., Frenklach M. Graphene layer growth chemistry: Five- and six-member ring flip reaction. J Phys Chem A. 2008;112(10):2125-30.

[28] Whitesides R, Kollias AC, Domin D, Lester Jr WA, Frenklach M. Graphene layer growth: Collision of migrating five-member rings. Proc Combust Inst. 2007;31:539-46.

[29] Whitesides R, Frenklach M. Detailed kinetic Monte Carlo simulations of graphene-edge growth. J Phys Chem A. 2010;114(2):689-703.

[30] Raj A, Man PLW, Totton TS, Sander M, Shirley RA, Kraft M. New polycyclic aromatic hydrocarbon (PAH) surface processes to improve the model prediction of the composition of combustion-generated PAHs and soot. Carbon. 2010;48(2):319-32.

[31] You X, Whitesides R, Zubarev D, Lester Jr WA, Frenklach M. Bay-capping reactions: kinetics and influence on graphene-edge growth. Proc Combust Inst. 2011;33:685-92.

[32] Sendt K, Haynes BS. Density functional study of the chemisorption of $\mathrm{O}_{2}$ on the armchair surface of graphite. Proc Combust Inst. 2005;30(2):2141-9.

[33] Sendt K, Haynes BS. Density functional study of the chemisorption of $\mathrm{O}_{2}$ on the zig-zag surface of graphite. Combust Flame. 2005;143(4):629-43.

[34] You X, Zubarev DY, Lester WA, Jr., Frenklach M. Thermal decomposition of pentacene oxyradicals. J Phys Chem A. 2011;115(49):14184-90.

[35] Zubarev DY, Robertson N, Domin D, McClean J, Wang J, Lester WA, Jr., et al. Local electronic structure and stability of pentacene oxyradicals. J Phys Chem C. 2010;114(12):5429-37.

[36] Edwards DE, You X, Zubarev DY, Lester Jr WA, Frenklach M. Thermal decomposition of graphene armchair oxyradicals. Proc Combust Inst. 2013;34:1759-66.

[37] Edwards DE, Zubarev DY, Lester WA, Frenklach M. Pathways to soot oxidation: Reaction of OH with phenanthrene radicals. J Phys Chem A. 2014;118(37):8606-13.

[38] Zhou C-W, Kislov VV, Mebel AM. Reaction mechanism of naphthyl radicals with molecular oxygen. 1. Theoretical study of the potential energy surface. J Phys Chem A. 2012;116(6):1571-85.

[39] Kislov VV, Singh RI, Edwards DE, Mebel AM, Frenklach M. Rate coefficients and product branching ratios for the oxidation of phenyl and naphthyl radicals: A theoretical RRKM-ME study. Proc Combust Inst. 2015;35:1861-9.

[40] Singh RI, Mebel AM, Frenklach M. Oxidation of graphene-edge six- and five-member rings by molecular oxygen. J Phys Chem A. 2015;119:7528-47.

[41] Raj A, da Silva GR, Chung SH. Reaction mechanism for the free-edge oxidation of soot by $\mathrm{O}_{2}$. Combust Flame. 2012;159:3423-36.

[42] $\mathrm{Xu} \mathrm{K}, \mathrm{Ye} \mathrm{PD}$. Theoretical study on the oxidation mechanism and dynamics of the zigzag graphene nanoribbon edge by oxygen and ozone. J Phys Chem C. 2014;118(19):10400-7.

[43] Hernández-Giménez A, Castelló D, Bueno-López A. Diesel soot combustion catalysts: review of active phases. Chem Pap. 2014;68(9):1154-68.

[44] Whitesides R, Frenklach M. Effect of reaction kinetics on graphene-edge morphology and composition. Z Phys Chem. 2014;229:597-614.

[45] Gillespie DT. Exact stochastic simulation of coupled chemical reactions. J Phys Chem 1977;81(25):2340-61.

[46] Gillespie DT. Markov processes: An introduction for physical scientists. San Diego, CA: Academic Press; 1992.

[47] Frenklach M. Monte Carlo simulation of diamond growth by methyl and acetylene reactions. J Chem Phys. 1992;97:5794-802. 
[48] Frenklach M. Monte Carlo simulation of hydrogen reactions with the diamond surface. Phys Rev B. 1992;45:9455-8.

[49] Radovic LR, Silva-Villalobos AF, Silva-Tapia AB, Vallejos-Burgos F. On the mechanism of nascent site deactivation in graphene. Carbon. 2011;49:3471-87.

[50] Neoh KG, Howard JB, Sarofim AF. Soot oxidation in flames. In: Siegla DC, Smith GW, eds. Particulate Carbon: Formation During Combustion. New York: Plenum 1981, p. 261-82.

[51] Neoh KG, Howard JB, Sarofim AF. Effect of oxidation on the physical structure of soot. Proc Combust Inst. 1985;20:951-7.

[52] Sirignano M, Kent J, D'Anna A. Modeling formation and oxidation of soot in nonpremixed flames. Energy Fuels. 2013;27:2303-15.

[53] Allinger NL, Yuh YH, Lii JH. Molecular mechanics - The MM3 force-field for hydrocarbons. 1. J Am Chem Soc. 1989;111(23):8551-66.

[54] Ponder JW. TINKER: Software tools for molecular design. 4.2 ed. Washington University School of Medicine: Saint Louis, MO 2004.

[55] Murry RL, Colt JR, Scuseria GE. How accurate are molecular mechanics predictions for fullerenes? A benchmark comparison with Hartree-Fock self-consistent field results. J Phys Chem. 1993;97(19):4954-9.

[56] Schulman JM, Disch RL. Bowl-shaped hydrocarbons related to C-60. J Comput Chem. 1998;19(2):189-94.

[57] Frenklach M, Ebert LB. Comment on the proposed role of spheroidal carbon clusters in soot formation. J Phys Chem. 1988;92(2):561-3.

[58] Frenklach M, Clary DW, Gardiner WC, Jr., Stein SE. Detailed kinetic modeling of soot Formation in shocktube pyrolysis of acetylene. Proc Combust Inst. 1985;20:887-901.

[59] Brezinsky K. The high-temperature oxidation of aromatic hydrocarbons. Prog Energy Combust Sci. 1986;12:1-24.

[60] Thomas JM, Hughes EEG. Localized oxidation rates on graphite surfaces by optical microscopy. Carbon. 1964;1:209-14.

[61] Acik M, Chabal YJ. Nature of graphene edges: A Review. Jap J Appl Phys. 2011;50:070101.

[62] Fang HL, Lance MJ. Influence of soot surface changes on DPF regeneration. SAE Technical Paper 2004-013043, 2004.

[63] Suuberg EM. Thermally induced changes in reacivity of gases. In: Lahaye J, Ehrburger P, eds. Fundamental Issues in Control of Carbon Gasification Reactivity. Dordrecht: Kluwer Academic 1991, p. 269-305.

[64] Salatino P, Senneca O, Masi S. Assessment of thermodeactivation during gasification of a bituminous coal char. Energy Fuels. 1999;13:1154-9. 